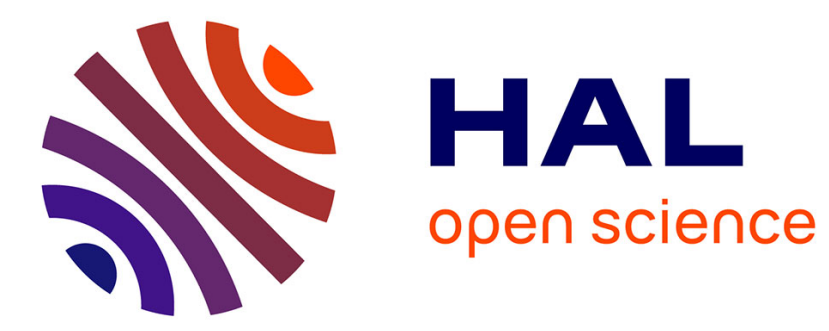

\title{
Performance Measurement of Mobile Manipulators
}

Roger Bostelman, Tsai Hong, Jeremy Marvel

\section{To cite this version:}

Roger Bostelman, Tsai Hong, Jeremy Marvel. Performance Measurement of Mobile Manipulators.

SPIE - DDS 2015, Apr 2015, Baltimore, Maryland, United States. hal-01401578

\section{HAL Id: hal-01401578 \\ https://hal.science/hal-01401578}

Submitted on 23 Nov 2016

HAL is a multi-disciplinary open access archive for the deposit and dissemination of scientific research documents, whether they are published or not. The documents may come from teaching and research institutions in France or abroad, or from public or private research centers.
L'archive ouverte pluridisciplinaire HAL, est destinée au dépôt et à la diffusion de documents scientifiques de niveau recherche, publiés ou non, émanant des établissements d'enseignement et de recherche français ou étrangers, des laboratoires publics ou privés. 


\title{
Performance Measurement of Mobile Manipulators
}

\author{
Roger Bostelman ${ }^{1}$ \\ National Institute of Standards and Technology, Engineering Laboratory, Intelligent Systems \\ Division, 100 Bureau Drive, MS8230, Gaithersburg, MD 20899 \\ IEM, Le2i, Université de Bourgogne,BP 47870, 21078 Dijon, France \\ Tsai Hong, Jeremy Marvel \\ National Institute of Standards and Technology, Engineering Laboratory, Intelligent Systems \\ Division, 100 Bureau Drive, MS8230, Gaithersburg, MD 20899
}

\begin{abstract}
This paper describes a concept for measuring the reproducible performance of mobile manipulators to be used for assembly or other similar tasks. An automatic guided vehicle with an onboard robot arm was programmed to repeatedly move to and stop at a novel, reconfigurable mobile manipulator artifact (RMMA), sense the RMMA, and detect targets on the RMMA. The manipulator moved a laser retroreflective sensor to detect small reflectors that can be reconfigured to measure various manipulator positions and orientations (poses). This paper describes calibration of a multi-camera,motion capture system using a 6 degree-of-freedom metrology bar and then using the camera system as a ground truth measurement device for validation of the reproducible mobile manipulator's experiments and test method. Static performance measurement of a mobile manipulator using the RMMA has proved useful for relatively high tolerance pose estimation and other metrics that supportstandard test method development for indexed and dynamic mobile manipulator applications.
\end{abstract}

Keywords: mobile manipulator, reproducible performance, smart manufacturing, ground truth, test methods, artifact

\section{INTRODUCTION}

Future smart manufacturing systems will include more complex coordination of mobile manipulators (i.e., robot arms mounted on mobile bases). "Mobile manipulators offer high mobility and manipulability. An ideal utilization of the motion redundancy in the mobile manipulator is to perform assembly tasks on a moving vehicle body while tracking."'[1] Mobile base and onboard manipulator motion may or may not be known a priori for applications such as assembly or even welding. However, performance of the combined mobile manipulator system must be known. Standard mobile manipulator performance has not been measured due to lack of standard test methods.

Historically, mobile manipulator research has focused on control algorithms, including dynamic motions caused by the onboard manipulator motion, especially in undulating terrain (e.g., outdoors [2]). Other areas of mobile manipulator research have been application-oriented, such as trajectory planning for a mobile manipulator with stability considerations and task planning, such as opening doors. [3] However, generalized measurement of mobile manipulators performing routine tasks (e.g., moving to different poses and generating motion profiles) has been relatively non-existent except for simply ensuring that the task is more or less completed. Prior to industrial acceptance for mobile manipulators, just as with their base components of industrial robot arms and automated guided vehicles (AGVs), these new mobile manipulator systems require their manufacturers to have real, verifiable performance data so users can apply these technologies appropriately.

The ISO 9283:1998 [4],industrial robot performance standard, describes accuracy and repeatability testing for fixedbase industrial robots for both end-of-motion poses and in-transit trajectories. Currently, there are no industrial robot performance standards for when industrial robots are onboard a moveable base. A standards committee

\footnotetext{
므oger.bostelman@nist.gov; phone 1-301-975-3426; fax 1-301-990-9688
} 
forAGV performance standard, ASTM F45 Committee on Driverless Automatic Industrial Vehicles [5],has recently been formed and mayinclude some aspect of mobile manipulation, e.g., docking.

Metrology methods for measuring performance of mobile manipulators, used to access parts or assemblies in manufacturing processes, include: physical contact using a touch probe [6], cameras detecting fiducials [7],laser interferometry, theodolites, and coordinate measuring arms [8]. An example uncertainty (position accuracy) for the camera calibration system in [8] was $0.1 \mathrm{~mm}$ and $0.2 \mathrm{~mm}$ for $650 \mathrm{~mm}$ and $950 \mathrm{~mm}$ target distances, respectively.[9] Other metrology methods inaccordance with ISO 9283 include:path comparison, trilateration, polar coordinate measuring, triangulation, optical tracking, inertial measuring, Cartesian coordinate, and path drawing. An alternative to these methodsis a conceptually simple and relatively inexpensive performance measurement method that could be replicated and used by mobile manipulator manufacturers and users.

Measurement of the robot's Cartesian position and orientation (pose), which is combined with the mobile base's pose, is complex since the system can include nine or more degrees of freedom. To simplify measurement, an artifact was designed to allow various geometric patterns to be traced by the robot wielding a tool point sensor to sense the dimensional points along the patterns. As a starting point, the AGV with onboard robot arm was moved to a series of locations along a straight linein order to measureits uncertainty from various directions. For example, it has been shown in previous research [10] that an AGV approaching the same point from various directions is relatively inaccurate as compared to approaching from the same direction. In a flexible manufacturing environment, this may be an issue where the manipulator might not be able to access the parts or assembly due to AGV position uncertainty.

This paper discusses a reconfigurable mobile manipulator artifactconcept, experiments, and results for measuring performance of a mobile manipulator. Calibration of the ground truth system used to measure uncertainty of a component (e.g., AGV, manipulator, artifact) is first described to provide a sound basis for the concept. With the artifact method, repeatability and accuracy of a mobile manipulator can then be measured for three main scenarios: A) the AGV stops while the robot accesses all points within its work volume, B) the AGV initially stops while the robot accesses all or most points within its work volume, as well as informs the AGV to increment to a new point and stop while the robot accesses additional points, and C) both the AGV and robot simultaneously move while the robot accesses all points. This paperwilldetail experiments and results of performing scenario A. Scenario B is an extension of scenario A, which uses similar testing to measure uncertainty (for example, the AGV simply moves and stops at a different location). Scenario $\mathrm{C}$ is much more complex, since coordination of both the AGV and manipulator is needed and requires timely communication between the two systems. Therefore, scenario $\mathrm{C}$ is left to future research, with the described scenarios used as a basis for its execution.

\section{PERFORMANCE MEASUREMENT CONCEPT}

The concept utilizes a reconfigurable mobile manipulator artifact (RMMA) (see Figure 1) that can provide a desired measurement uncertainty as a comparisonto ground truth (GT).The RMMAcan be in a horizontal, vertical, or other known orientation, as would be typical of assembly in manufacturing. With the RMMA, manipulator pose (i.e., position and orientation) is measured as a digital detect or no detect at a specified distance and angle from the task point. For our tests, detector distance above the reflector was approximately $127 \mathrm{~mm}$. The distance would be representative of a programmed waypoint above and in-line with the next robot task point to grip or insert a part or perform another task. The desired uncertainty may be, for example, apart insertion alignment tolerancerequired for a manufacturing assembly process. Moving along this grip/insertion line, at the aligned pose to the task point, also provides some knowledge of insertion performance (i.e., if the task point is continuously detected along the grip/insertion line) although this measurement is for future research. The RMMAis constructed of anodized, machined aluminum, and has a series of tapped holes of known geometric patterns and position tolerance of \pm 0.01 $\mathrm{mm}$. The patterns include a circle, square, triangle, straight and curved lines, sinusoids, etc. Each adapter, to be screwed into the various patterns of holes, supports a background target, a circular reflector, and a cylinder used as a light collimator. Flat, background targets, measuring $7.6 \mathrm{~cm}$ diameter and $6.4 \mathrm{~mm}$ incremental rings are perpendicular to each collimator as a simple visual cue for the test director when the robot does not align with the reflector. The adapter, reflector,target, and collimator can be perpendicular to the flat surface or rotated to pitch angles between $\pm 90^{\circ}$ and yaw angles between $0^{\circ}$ and $360^{\circ}$. The reflector and the collimatorinsidecan be any diameter, dependent upon the sensor specification and the desired measurement uncertainty. 


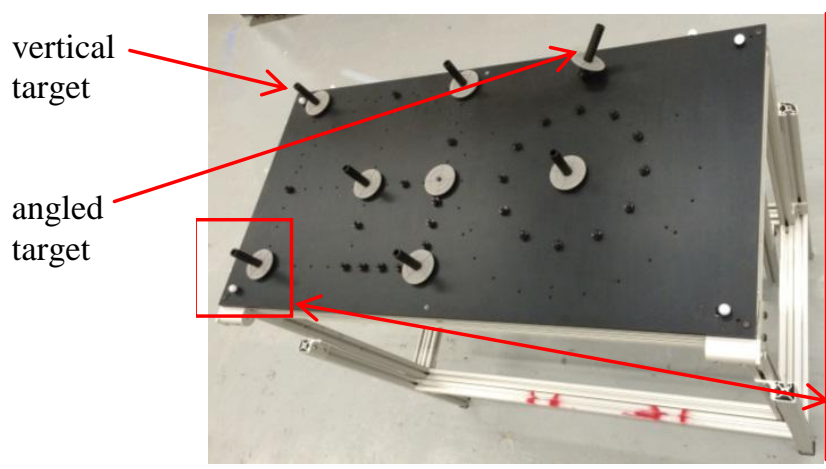

a

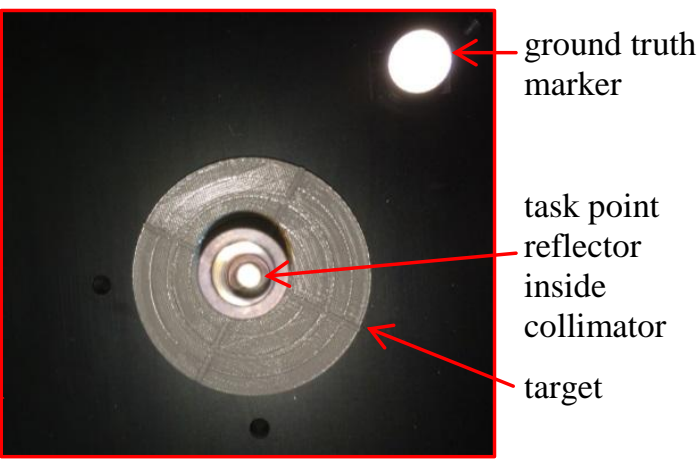

$\mathrm{b}$

Figure 1 - (a) Reconfigurable mobile manipulator artifact, (b) Close-up, top view of an illuminated reflector inside a collimator and an illuminatedground truth marker.

The RMMA can be horizontal (as tested in this research), angled, or vertical configurations, at short-to-tall heights.It can also be above the mobile manipulator for an unlimited set of performance measurement possibilities. Figure 2 shows the horizontal artifact experimental configuration and the $457.20 \mathrm{~mm}$ square pattern used for our experiment.

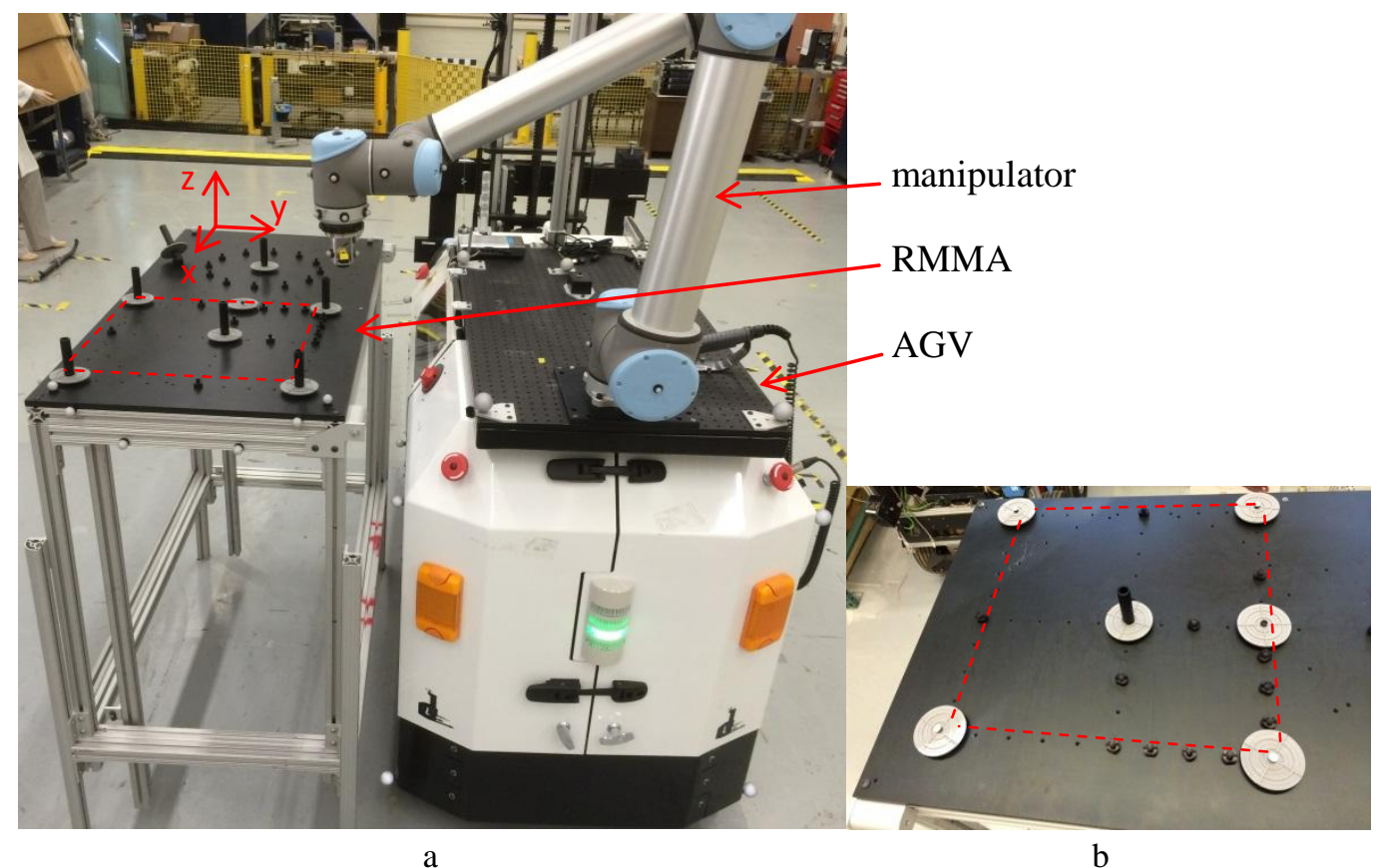

Figure 2 - (a) Test configuration showing the mobile manipulator beginning the performance measurements. (a, b) The manipulator test pattern is shown with red dashed lines between four vertices of a $457.20 \mathrm{~mm}$ square. (b) The same square as in (a) setup with larger markers and no light collimators.

The performance measurement concept uses a retroreflective laser sensor (RLS, that provides a relatively narrow beam consisting of both an emitting laser and adetector to detect $6.4 \mathrm{~mm}$ diameter reflectors (i.e., task points) positioned in known patterns (e.g., square, circle, triangle, sinusoids, etc.). A camera, with a light source, could instead be used as the detection sensor, especially with a larger diameter reflector or other spot.The reflectorscan have specific diameters depending upon the required uncertainty for their location. The manipulator ${ }^{2}$ used has a manufacturer's specified repeatability of $0.1 \mathrm{~mm}$. [11]The RLS, shown in Figure 3 (a),shows the sensormounted inline and perpendicular to the manipulatortool point. Initial alignment to the reflector can occur using one of several

\footnotetext{
${ }^{2}$ Disclaimer: Commercial equipment and materials are identified in order to adequately specify certain procedures. In no case does such identification imply recommendation or endorsement by the National Institute of Standards and Technology, nor does it imply that the materials or equipment identified are necessarily the best available for the purpose.
} 
methods briefed in Section 1, Introduction. For our experiments, we chose to release the manipulator brakes and manually position the RLS until all reflectors at the four locations were detected by the laser. During the experiment, the manipulator began a spiral searchpattern to detect the reflectors beginning with the manually taught positions. Each search pattern was parameterized to search in a growing, 15-spiral pattern with a maximum radius of $50 \mathrm{~mm}$; the trajectory took two minutes and would be interrupted when the reflector was detected. When a reflector was detected, the manipulator received a digital signal change and the manipulator was commanded to move to the next taught reflector location and begin another spiral search, and so on. If no detector was detected, the spiral search pattern was executed.If there was still no detection within $2 \mathrm{~min}$, the manipulator logged a "failed" and moved to the next reflector location. If detected, the manipulator logged a "success" and moves to the next location, and so on.

Without the collimator, as shown in Figure 2 (b), the reflector can be detected at approximately $\pm 20^{\circ}$ to the vertical axis. For the collimators used, the reflector can be detected: 1) at a maximum $3.2 \mathrm{~mm}$ radius from the reflector center; 2) at approximately $\pm 7^{\circ}$ to the vertical axis (see Figure 3 (b));3) when at the closest RLS detect position at $10.2 \mathrm{~cm}$ minimum above the reflector; and 4) when using a $12.7 \mathrm{~mm}$ inside-diameter collimator. Collimators could be made with even smaller inside-diameters to force more perpendicular robot tool point axis pose to the reflector. The sensor used was specified to detect a minimum $4 \mathrm{~mm}$ diameter reflector repeatedly at a distance of $10 \mathrm{~cm}$.
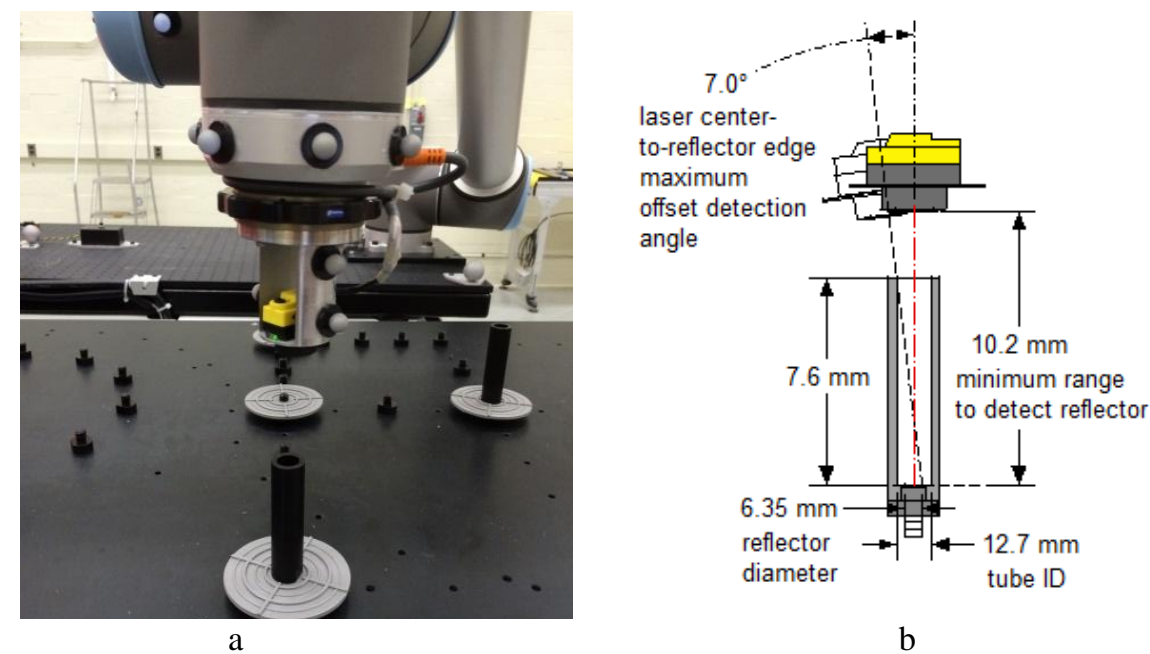

Figure 3-Retroreflective lasersensor assembly: a laser retroreflector (yellow and black) attached to a robot adapter. Spherical markers are also shown attached to the RLS, robot, tool-mount, and the AGV (background), as well as to the RMMA surface (not shown). (b) RLS measurement distances and offsets.

\section{EXPERIMENTS AND RESULTS}

A multi-camera ground truth (GT) system was setup in a National Institute of Standards and Technology (NIST) lab. Twelve cameras were mounted to the four lab walls at a height of $4.3 \mathrm{~m}$ above the floor and set to view the area where the experiments were performed. Several variables were added to the mobile manipulator position measurement uncertainty, beginning with the GT system. Three types of experiments were performed to quantize these variables, including: 1) comparison ofthe multi-camera GTmeasurement system [12] uncertainty to a metrology bar, 2) comparison of the AGV and RMMA static position to GT, and 3)comparison of a mobile manipulatoruncertainty to the RMMA and to GT.

For experiment 1, the metrology bar (shown in Figure 4 (a)), assembled at NIST with known dimensions, was used to test the uncertainty of the GT throughout the $9 \mathrm{~m}$ x $22 \mathrm{~m}$ AGV lab. The bar was positioned and then moved throughout the space where the AGV was to be used. The GT system measured the distance between each bar end with five markers. [13]Analysis of data provides bar length measurement uncertainty throughout the space. Uncertainty is based on the [14] describing standard deviation ( $\sigma)$ as $68 \%$ confidence. Measurement $\sigma$ of the static uncertainty is $0.022 \mathrm{mmand}$ static angle is $0.023^{\circ}$. The dynamic distance uncertainty (i.e., the positional accuracy) is 
$0.26 \mathrm{~mm}$, and $\sigma$ of the angle is $0.10^{\circ}$. Figure 4 (b) is a screenshot of the GT display for the remaining experiments showing the cameras, AGV, manipulator, and the RMMA objects being tracked. The markers on each component are grouped togetherso that each component motion can be analyzed independent from one another. For example, the AGV markers are grouped separate from the manipulator markers, etc.,as depicted by the different colors.

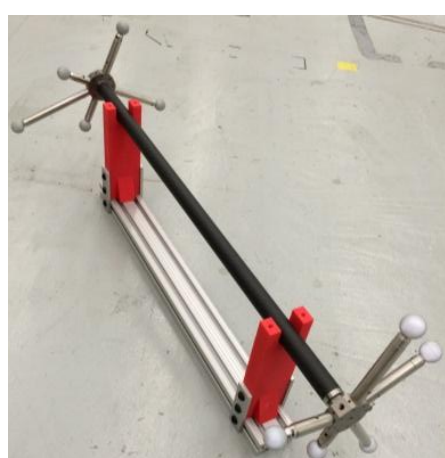

a

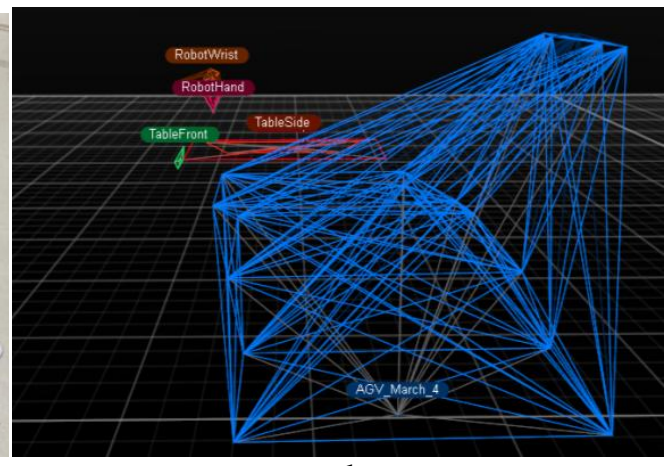

b

Figure 4 - (a) NIST metrology bar used to measure ground truth system uncertainty. (b) Screen capture from the multi-camera ground truth system showing the cameras, AGV, manipulator, and the RMMA.

Experiment 2 included three tests: A) measure the AGV position near the RMMA relative to the commanded AGV position, B) measure the AGV static position movement relative to GT, and C) measure the RMMA static movement relative to GT. We first considered a non-ideal case, perhaps typical of industry, for the AGV calibration. The AGV is approximately two years old and has not been calibrated since purchase. The AGV was moved from a home position approximately $5 \mathrm{~m}$ to the artifact location and back to home 10 times and was repeated three times. Table 1 shows uncertainty of AGV commanded versus AGV tracked data for a single stop point. The average offset is $29 \mathrm{~mm}$ in $\mathrm{X}$ and $-9 \mathrm{~mm}$ in Y. Compensation for this offset was performed during test setup of the initial robot positioning over the first-accessed reflector location. Table 2 displays the uncertaintyin the distancebetween the RMMA originand the AGV origin in the table plane. Table 2 shows that the expected AGV stop position near the RMMA has a fairly repeatable average offset.

Table 1 - Difference (in mm) of the commanded AGV versus AGV tracked data for the AGV stop point beside the RMMA.

\begin{tabular}{|c|c|c|c|}
\hline Axis & Run1 & Run2 & Run3 \\
\hline $\mathrm{X}$ & 27 & 29 & 30 \\
\hline $\mathrm{Y}$ & -7 & -10 & -9 \\
\hline
\end{tabular}

Table 2. Uncertainty(in mm) in the distancebetween the RMMA originand the AGV origin in the RMMA plane.

\begin{tabular}{|l|l|l|l|}
\hline & Run1 & Run2 & Run3 \\
\hline Distance & 0.095 & 0.088 & 0.090 \\
\hline
\end{tabular}

Experiment 2, test $\mathrm{B}$ and test $\mathrm{C}$ measure theuncertainty of two more variables beyond the AGV position, including the movement of each component, AGV, and RMMA, at the AGV static positions. It might be expected that the relatively heavy AGV (approximately $1137 \mathrm{~kg}$ ), with respect to the onboard lightweight manipulator $(29 \mathrm{~kg})$, does not move. In addition, the one story, $7 \mathrm{~m}$ high lab is located on a ground level with concrete floor, yet there is GTmeasured building movement that may affect precision assembly performance of a mobile manipulator. Our lab is perhaps evenmore ideal than typical factory settings where many more machines and processes could cause even larger measurement-disturbing vibrations than our experiments uncovered. Figures 5 (a) and (b), show GT point cloudsof the AGV movement,when it was stopped beside the RMMA and while themanipulator accesses each location, and of the RMMA movement, respectively. Tables 3 and 4 listthe mean $(\mu)$ and standard deviation $(\sigma)$ of position (in $\mathrm{mm}$ ) uncertainty with respect to the GT origin for each of the three runsfor the AGV and RMMA, respectively. Although the mean is relatively small for both the AGV and the RMMA, the movement spans from approximately $0.15 \mathrm{~mm}$ in $\mathrm{X}$ and $0.25 \mathrm{~mm}$ in $\mathrm{Y}$ for the AGV, and $0.5 \mathrm{~mm}$ in $\mathrm{X}$ and $0.6 \mathrm{~mm}$ in $\mathrm{Y}$ for the RMMA. Independently, or worse, combining these maximum uncertainties $(0.52,0.65)$ could induce enough position offset 
from the reflector to cause the manipulator to begin a search at each location since the initial spiral search provides reflector detection by the RLS at the reflector edge and not at the center.

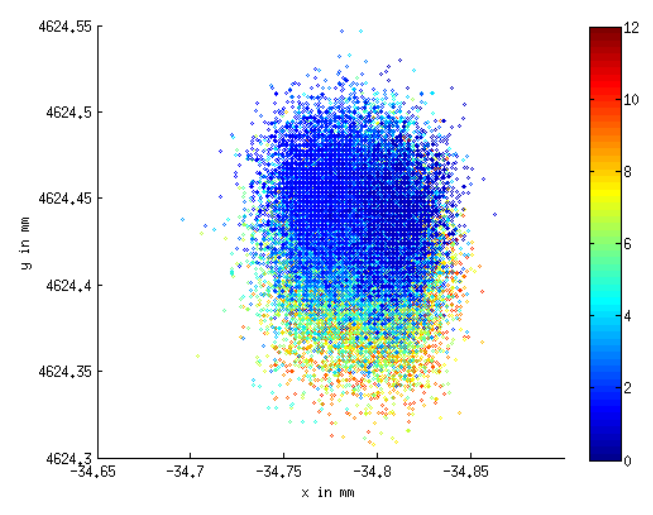

a

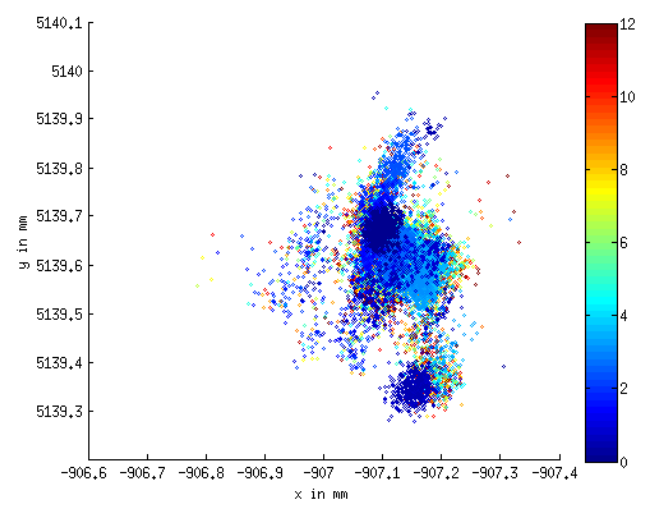

b

Figure 5 - GT data points relative to its origin (in $\mathrm{mm}$ ) of (a) the stationary AGV and (b) the RMMAmovementover time (in minutes), shown by the varying colors, while the manipulator moves the RLS attempting to detect RMMA reflectors.

Table 3 - GT measured distances, with respect to the GT origin, showing the AGV movement when stopped beside the RMMA and while the RLS attempts to detect RMMA reflectors.

\begin{tabular}{|c|c|c|c|c|c|c|}
\hline & \multicolumn{2}{|c|}{ Run1 } & \multicolumn{2}{c|}{ Run2 } & \multicolumn{2}{c|}{ Run3 } \\
\hline Axis & $\mu$ & $\sigma$ & $\mu$ & $\sigma$ & $\mu$ & $\sigma$ \\
\hline $\mathrm{X}$ & -34.79 & 0.021 & -34.67 & 0.028 & -34.13 & 0.027 \\
\hline $\mathrm{Y}$ & 4624.43 & 0.032 & 4626.50 & 0.032 & 4626.0 & 0.042 \\
\hline
\end{tabular}

Table 4 - GT measured distances, with respect to the GT origin,of the RMMA movement

\begin{tabular}{|c|c|c|c|c|c|c|}
\hline & \multicolumn{2}{|c|}{ Run1 } & \multicolumn{2}{c|}{ Run2 } & \multicolumn{2}{c|}{ Run3 } \\
\hline Axis & $\mu$ & $\sigma$ & $\mu$ & $\sigma$ & $\mu$ & $\sigma$ \\
\hline $\mathrm{X}$ & -907.13 & 0.040 & -907.13 & 0.043 & -907.13 & 0.041 \\
\hline $\mathrm{Y}$ & 5139.67 & 0.15 & 5139.59 & 0.099 & 5139.61 & 0.085 \\
\hline
\end{tabular}

Experiment 3 included threerunsto measure position uncertainty of the manipulator relative to the RMMA and to GT.Each trial consisted of 33 repetitions of a cyclic search for four reflectors with known characteristics. At each reflector, the manipulatorinitiated a spiral search to locate the reflectorwith the RLS. The first cycle allowed the manipulator to locate all reflectors followed by 32 cycles to measure repeatability.

Each runconsisted of the AGV moving to the registration pose from an initial "home" position, thus incurring some position and orientation error at the registration point. Once at the registration point, a beam break sensor attached to the AGV signaled the manipulator to initiate the registration process. The manipulatormoved to the pose where it expected to find the first registration point, $p_{1}$, and began the spiral pattern. Once the reflector was detected, the position of the reflectorwas recorded as $p_{1}^{\prime}$, and the manipulatormoved to where it was expected to find the second registration point, $\tilde{p}_{2}$, based on the nominal distance offset and orientation expectations from $p_{1}^{\prime}$ defined prior to the trial. At $\tilde{p}_{2}$, the manipulatorperformed the spiral search again until the reflectorwas found, and then recorded the reflector's position, $p_{2}^{\prime}$. The manipulatorthen updated its expectation of the artifact's position and orientation based on the Euler angle of incidence between $p_{1}^{\prime}$ and $p_{2}^{\prime}$, and moves to the new estimate for the location of the third registration point, $\tilde{p}_{3}$. The spiral search is again repeated until the reflectoris located, the manipulator control program recorded its position as $p_{3}^{\prime}$, and the manipulator performed one last spiral search at the fourth registration point, $\tilde{p}_{4}$. This search process is illustrated in Figure6. 


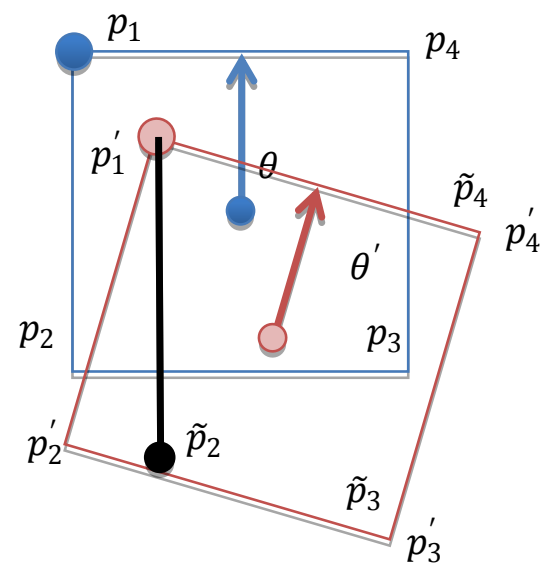

Figure 6 - Comparison of the nominal (blue) and measured (red) poses of the registration artifact reflectors after the first search repetition. The expected positions for $p_{2}$ through $p_{4}$ are updated based on the measurements of the prior target locations.

At this point, all four registration points were updated with their actual poses, and the process was then repeated 32 more times using $p_{1}^{\prime}$ through $p_{4}^{\prime}$ as initial search locations to capture positional uncertainty and repeatability measurements. Once the $33^{\text {rd }}$ iteration was complete, the manipulatorsignaled to the AGV that the manipulator had been registered with updated position (X-Y plane) and orientation ( $\mathrm{Z}$ rotation) offsets. The AGV then moves back to its home position, and the process repeated. Figure 7 (a) shows the GT data at each of four locations and a closeup of location 1 data in Figure 7 (b) for Run 1 for 33 times over approximately 10 min as expressed by the color bar.
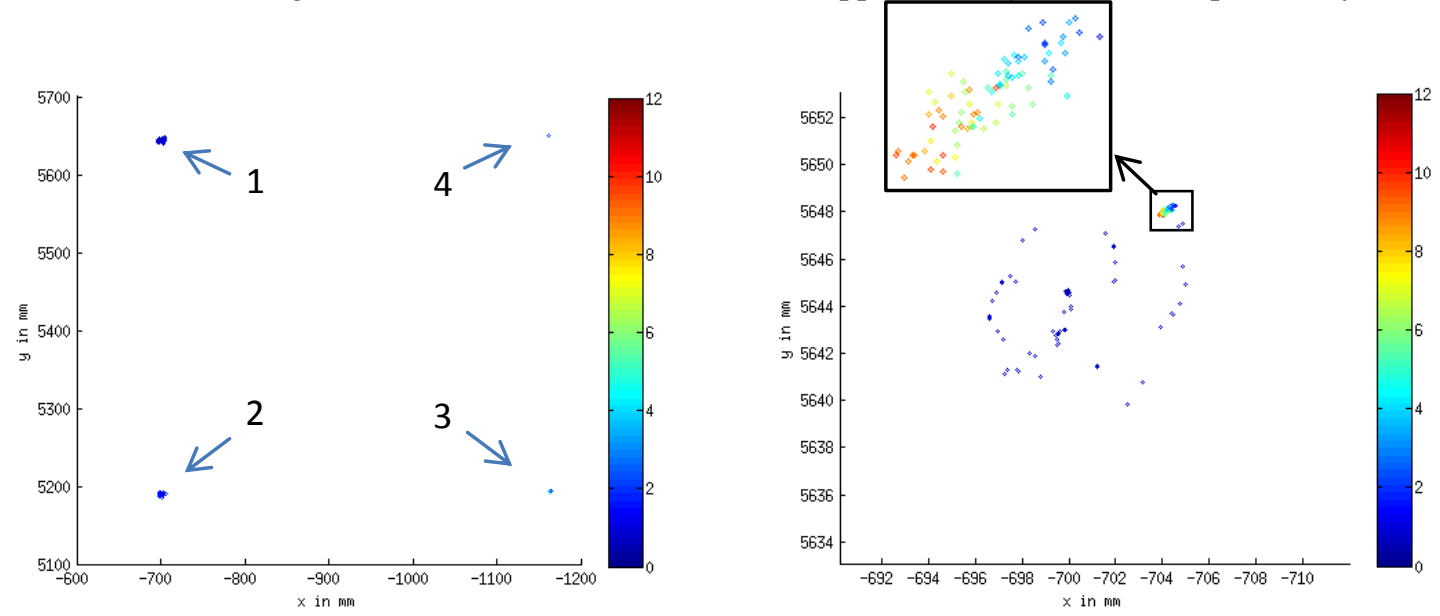

Figure 7 - (a) GT data of time at each of four locations in Run 1 for 33 times over approximately 10 min as expressed by the color bar. (b) Close-up showing the first spiral search at location 1 beginning Run 1 and no search detect (inset) at location 1 for the remaining 32 times.

The close-up clearly shows data points plotted in curved lines from the first spiral search at location 1 at the beginning of Run 1 . Then, no search was used detect location 1 for the remaining 32 times as depicted by the multicolored data points in a much tighter data pattern which spans approximately $0.8 \mathrm{~mm}$ in $\mathrm{X}$ and $0.5 \mathrm{~mm}$ in $\mathrm{Y}$. The plotted data is from only when the manipulator has a velocity of less than $0.1 \mathrm{~mm} / \mathrm{s}$. Note the larger data spots shown in Figure 7 (a) for locations 1 and 2 compared to locations 3 and 4 proving that the mobile manipulator has registered pose with the RMMA.

Table 5 shows the mean mobile manipulator position when the RLS detected the reflector for the remaining 32 time process, and for the number of thresholded points, as compared to each reflector center. Since the manipulator almost continuously moves, perhaps even when the reflector is detected (e.g., when the laser moves across the 
detector), displayed in the table is a mean uncertainty of the RLS position compared to the GT reflector position center for all three runs.

Table 5 - Actual reflector point locations and mean mobile manipulator positions when the RLS detected the reflector for the remaining 32 timesas compared to each reflector center.

\begin{tabular}{|c|c|c|c|}
\hline \multirow{2}{*}{} & \multicolumn{3}{|c|}{$\begin{array}{c}\text { Mean position (mm) relative to } \\
\text { reflector center }\end{array}$} \\
\cline { 2 - 4 } & Run 1 & Run 2 & Run 3 \\
\hline \# points & 26 & 74 & 41 \\
\hline Location 1 & \multicolumn{3}{|}{} \\
\hline$x(\mathrm{~mm})$ & -2.61 & -2.57 & -2.35 \\
\hline$y(\mathrm{~mm})$ & 1.09 & 0.05 & 0.83 \\
\hline Location 2 & -1.88 & -1.74 & 1.46 \\
\hline$x(\mathrm{~mm})$ & \multicolumn{3}{|l}{} \\
\hline$y(\mathrm{~mm})$ & 0.50 & 0.28 & -0.73 \\
\hline Location 3 & \multicolumn{3}{|l}{} \\
\hline$x(\mathrm{~mm})$ & -1.20 & -1.09 & -0.74 \\
\hline$y(\mathrm{~mm})$ & 1.29 & 0.94 & 0.69 \\
\hline Location 4 & \multicolumn{3}{|l}{} \\
\hline$x(\mathrm{~mm})$ & -1.74 & -1.81 & -1.08 \\
\hline$y(\mathrm{~mm})$ & 1.62 & 0.83 & -0.09 \\
\hline
\end{tabular}

After the thirdrun, the data logs from the manipulator were also analyzed to estimate the positional and orientation accuracy and repeatability performances of the AGV as a function of the registration artifact's nominal position and orientation. The orientation error was calculated as the difference between the expected orientation of the artifact, $\theta$, and the average calculated orientation, $\theta^{\prime}$, as determined by the angle of incidence between the calculated centroid of the artifact's four measured registration points ( $p_{1}^{\prime}$ through $p_{4}^{\prime}$ ), and the mid-point between the measured registration points $p_{1}^{\prime}$ and $p_{4}^{\prime}$. Two different methods were evaluated for calculating the position error. The first method simply compared the $\mathrm{X}$ and $\mathrm{Y}$ positional offsets of the centroid of the measured registration points with the centroid of the nominal registration point locations. The second method corrected for the orientation error about the centroid of the measured poses, and then compared the measured location of $p_{1}^{\prime}$ with the original nominal pose of $p_{1}$.

Two metrics were considered in our analysis: 1) pass/fail for initially detecting the reflector after the first search of all four reflectors and 2) the positional repeatability when the RLS detected the reflector. Table 6 shows results of the pass/fail test. Manipulator positioning was called a fail if after the first search trial and for the remaining 32 trials, the RLS did not detect a reflector immediately at each location requiring a search to begin.

Table 6-Pass/Fail and dwell time results for detecting the reflector without a search.

\begin{tabular}{|c|c|c|c|}
\hline & Run1 & Run2 & Run3 \\
\hline $\begin{array}{c}\text { Number Fails for each } \\
\text { position }(1,2,3,4)\end{array}$ & $1,1,0,0$ & $15,1,0,0$ & $1,1,5,1$ \\
\hline $\begin{array}{c}\text { Total search time }(s) \text { at } \\
\text { each location }(1,2,3,4)\end{array}$ & $41.5,42.9,0.0,0.0$ & $79.4,40.2,0.0,0.0$ & $41.6,78.1,38.6,31.2$ \\
\hline
\end{tabular}

Fails, therefore, correspond to the total search time that was required at each location. This metric could correspond to the time the manipulator would require, for example, to perform an assembly operation. Assembly times varied according to position uncertainty, part tolerances, initial pose error, search parameters, etc.

Results of the second metric are expressed in Table 7 for all runs and with respect to the robot and to GT. The robot uncertainty spanned between $0.01 \mathrm{~mm}$ and $0.74 \mathrm{~mm}$ in $X$ and between $0.11 \mathrm{~mm}$ and $0.42 \mathrm{~mm}$ in $\mathrm{Y}$ with orientation uncertainty between $-0.6^{\circ}$ and $-0.8^{\circ}$. However, orientation uncertainty, for these initial experiments, does not 
consider the measured reflector center (see Figure 8). Instead, as shown in Figure 8, RLS detection points may be at the upper and lower edges of location 1 and 2 reflectors, respectively, and cause large rotation error $\theta$ and also failed attempts on following RLS detect attempts.

Table 7 - Manipulator tool center point positional repeatability uncertainty, as logged by the manipulator and logged by the GT, when the RLS detected the reflector for the remaining 32 times.

\begin{tabular}{|c|c|c|c|}
\hline & \multicolumn{3}{|c|}{$\begin{array}{c}\text { Repeatability uncertainty } \\
\text { measured by the manipulator }\end{array}$} \\
\hline & Run 1 & Run 2 & Run 3 \\
\hline Location 1 & \multicolumn{3}{|l|}{} \\
\hline$x(\mathrm{~mm})$ & 0.15 & 0.74 & 0.11 \\
\hline$y(\mathrm{~mm})$ & 0.11 & 0.42 & 0.11 \\
\hline Location 2 & \multicolumn{3}{|l}{} \\
\hline$x(\mathrm{~mm})$ & 0.01 & 0.09 & 0.07 \\
\hline$y(\mathrm{~mm})$ & 0.27 & 0.18 & 0.28 \\
\hline Location 3 & \multicolumn{3}{|l|}{} \\
\hline$x(\mathrm{~mm})$ & 0.21 & 0.01 & 0.35 \\
\hline$y(\mathrm{~mm})$ & 0.12 & 0.28 & 0.16 \\
\hline Location 4 & \multicolumn{3}{|l|}{} \\
\hline$x(\mathrm{~mm})$ & 0.03 & 0.022 & 0.05 \\
\hline$y(\mathrm{~mm})$ & 0.27 & 0.28 & 0.27 \\
\hline
\end{tabular}

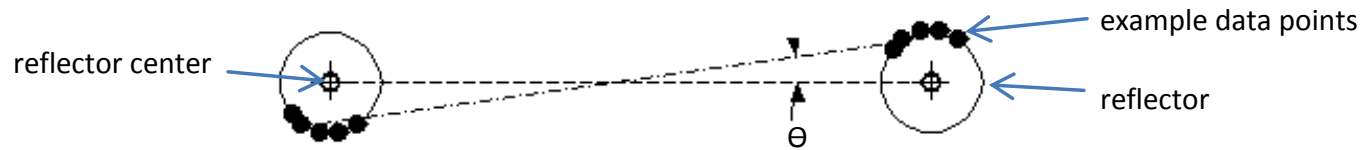

Figure 8 - Graphic of possible orientation uncertainty when the reflector center is not detected.

\section{ACKNOWLEDGEMENTS}

The authors would like to thank Sebti Foufou, Qatar University, Doha, Qatar for his guidance on this research.

\section{CONCLUSIONS}

This paper described a concept for measuring the reproducible performance of mobile manipulators to be used for assembly or other similar tasks. An AGV, with an onboard robot arm, was programmed to repeatedly move to and stop at a novel RMMA, sense the RMMA and the RLS, be repeatedly moved by the manipulator, and detect targets on the RMMA. Experiments were performed that tested a multi-camera motion capture system for use as ground truth for the mobile manipulator measurements and tested each component of the mobile manipulator, including the $\mathrm{AGV}$, the manipulator, and the RMMA. Initially, ground truth was established by comparing results of the system to a metrology bar of known length. Results showed that GT uncertaintyin static positional accuracywas $0.022 \mathrm{~mm}$ and uncertaintyof the orientation was $0.023^{\circ}$.

Further experiments proved that both the AGV and the RMMA were moving even with: the AGV stopped while the manipulator is moving, the AGV weight being nearly 40 times heavier than the manipulator, and the research lab having a concrete floor on the first building level. Results for the AGV and RMMA show that position uncertainty spans from approximately $0.15 \mathrm{~mm}$ in $\mathrm{X}$ and $0.25 \mathrm{~mm}$ in $\mathrm{Y}$ for the AGV and $0.5 \mathrm{~mm}$ in $\mathrm{X}$ and $0.6 \mathrm{~mm}$ in $\mathrm{Y}$ for the RMMA. When these uncertainties are combined, maximum uncertainties can be $(\mathrm{X}=0.52 \mathrm{~mm}, \mathrm{Y}=0.65 \mathrm{~mm})$ which could induce enough position offset to the reflector to cause the manipulator to begin a search at each location. Since the initial spiral search provides reflector detection by the RLS at the reflector edge and not at the center, the spiral search is perhaps not the complete performance test method. Instead, an additional raster scan of 
the $6.35 \mathrm{~mm}$ diameter reflector should follow the spiral search and the average detect position should be at the center of the reflector.

By detecting the reflector center, it is expected that the specified manipulator repeatability of $0.1 \mathrm{~mm}$ combined with the AGV and RMMA movements would provide increased detects at each reflector location after the initial scan, potentially without the need for a spiral search again until the AGV moves and returns to the same location. As measured by the manipulator, the largest maximum positional repeatability uncertainty was $(X=0.74 \mathrm{~mm}, \mathrm{Y}=0.42$ $\mathrm{mm}$ ) where adding the maximum AGV and RMMA movements uncertainties could be as high as $(\mathrm{X}=1.26 \mathrm{~mm}, \mathrm{Y}=$ $1.07 \mathrm{~mm}$ ). However, detection of the reflector edge potentially guarantees no better than $50 \%$ reflector detection since uncertainty could place the laser on or off the reflector. With respect to GT, the maximum uncertainty when adding $\mathrm{AGV}$ and RMMA movement to mean reflector detect position, compared to the reflector center, was $(\mathrm{X}=1.46$ $\mathrm{mm}+0.52 \mathrm{~mm}=1.98 \mathrm{~mm}, \mathrm{Y}=1.62 \mathrm{~mm}+0.65 \mathrm{~mm}=2.27 \mathrm{~mm}$ ) or $3.01 \mathrm{~mm}$ radius. Therefore, if the retroreflective laser sensor was initially positioned at the $6.4 \mathrm{~mm}$ diameter reflector center (i.e., $3.2 \mathrm{~mm}$ radius), the lasershould always detect the reflector. The test method used to measure the mobile manipulator performance appears appropriate with results that match system uncertainties and leave goal point search (e.g., raster scan to find the reflector center), reflector detect time, and perhaps other improvements to consider for future tests.

\section{REFERENCES}

[1] Bradley Hamner, Seth Koterba, Jane Shi, Reid Simmons, Sanjiv Singh, "Mobile Robotic Dynamic Tracking for Assembly Tasks," IROS 2009

[2] Hootsmans, N.A.M., Dubowsky, S., Mo, P.Z., "The experimental performance of a mobile manipulator control algorithm," Robotics and Automation, 1992. Proceedings, Proceedings, IEEE International Conference, 12-14 May 1992

[3] Roger Bostelman, Tsai Hong, Jeremy Marvel, "Survey of Research for Performance Measurement of Mobile Manipulators," Journal TBD, March 2015.

[4] International Standards Organization (ISO) 9283-1998 Manipulating industrial robots -- Performance criteria and related test methods, www.iso.org.

[5] ASTM F45 Committee on Driverless Automatic Industrial Vehicles, www.astm.org, 2014.

[6] MasterCal, http://www.americanrobot.com/products_mastercal.html,2005.

[7] C B. Atcheson, F. Heide, and W. Heidrich, "CALTag: High Precision Fiducial Markers for Camera Calibration," Vision, Modeling, and Visualization, 2010.

[8] Jose Mauricio, S. T. Motta, "Robot Calibration: Modeling Measurement and Applications," Industrial Robotics: Programming, Simulation and Applications, Low Kin Huat (Ed.), ISBN: 3-86611-286-6, InTech, 2006

[9] Kevin L. Conrad, Panayiotis S. Shiakolas, T. C. Yih, "Robotic Calibration Issues: Accuracy, Repeatability, and Calibration," Proceedings of the 8th Mediterranean Conference on Control \& Automation (MED 2000), Rio, Patras, Greece, 17-19 July 2000.

[10] Roger Bostelman, "Automatic Guided Vehicle (AGV) Performance Evaluation," unpublished research, NIST Engineering Laboratory Poster, 2014.

[11] Universal Robots A/S UR10 User Manual, UR10/CB3, Version 3.0 (rev. 15167), 2014.

[12] NaturalPoint, OptiTrack Motion Capture System, https://www.optitrack.com/, 2015.

[13] Roger Bostelman, Tsai Hong, and Gerry Cheok, "Navigation Performance Evaluation for Automatic Guided Vehicles,"7th IEEE International Conference on Technologies for Practical Robot Applications (TePRA), May 11-12, 2015.

[14] Taylor, B. N., and C. E. Kuyatt. "Guidelines for Evaluating and Expressing the Uncertainty of NIST Measurement Results-NIST Technical Note 1297, National Institute of Standards and Technology, Gaithersburg, MD." (1994). 